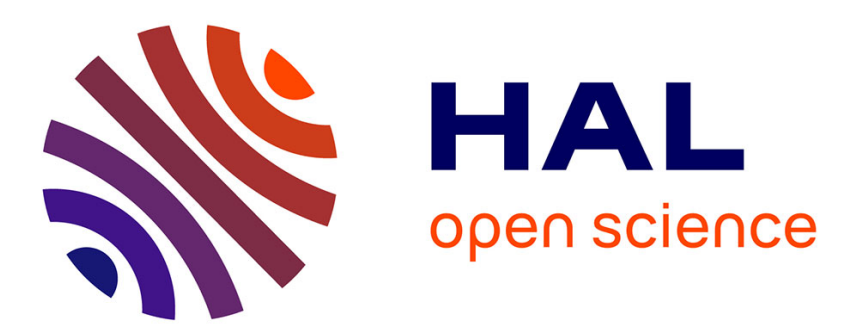

\title{
A methodology for assessing the vertical handover algorithms in heterogeneous wireless networks
}

Lucian Suciu, Karine Guillouard, Jean-Marie Bonnin

\section{To cite this version:}

Lucian Suciu, Karine Guillouard, Jean-Marie Bonnin. A methodology for assessing the vertical handover algorithms in heterogeneous wireless networks. BWAN'06: International workshop on broadband wireless access for ubiquitous networking, Sep 2006, Alghero, Sardinia, Italy. 10.1145/1189186.1189191. hal-02900754

\section{HAL Id: hal-02900754 https://hal.science/hal-02900754}

Submitted on 16 Jul 2020

HAL is a multi-disciplinary open access archive for the deposit and dissemination of scientific research documents, whether they are published or not. The documents may come from teaching and research institutions in France or abroad, or from public or private research centers.
L'archive ouverte pluridisciplinaire HAL, est destinée au dépôt et à la diffusion de documents scientifiques de niveau recherche, publiés ou non, émanant des établissements d'enseignement et de recherche français ou étrangers, des laboratoires publics ou privés. 


\section{A Methodology for Assessing the Vertical Handover Algorithms in Heterogeneous Wireless Networks}

\author{
Lucian Suciu, Karine Guillouard \\ France Télécom R\&D, Broadband Wireless Access Lab \\ BP59 4, rue de clos Courtel \\ 35512 Cesson-Sévigné, France \\ \{lucian.suciu, karine.guillouard\} \\ @francetelecom.com
}

\author{
Jean-Marie Bonnin \\ École Nationale Supérieure des Télécommunications \\ de Bretagne \\ CS 16607 - 2, rue de la Châtaigneraie \\ 35576 Cesson-Sévigné, France \\ jm.bonnin@enst-bretagne.fr
}

\begin{abstract}
The integration of heterogeneous access networks into a ubiquitous wireless environment has commenced. However, in contrast with homogeneous networks (e.g., GPRS or UMTS) where there are a lot of handover test-reference scenarios, extensive work has yet to be done for modelling the end-user mobility in heterogeneous wireless networks. This is partially due to the fact that thorough test-case emulations are complex in heterogeneous environments because the scenarios are difficult to put in practice; moreover, the handover algorithms' performances may also depend on the auxiliary mechanisms such as user profiling or decision parameters gathering. Therefore, in order to exclusively assess the vertical handover decision algorithms, we propose and develop a complete methodology and we investigate the associated mechanisms.
\end{abstract}

\section{INTRODUCTION}

The next-generation mobile wireless networks will give the end-user a greater choice of access technologies, and therefore, the decision to select the "best" interface and access network from many possible combinations has to be taken; this decision will depend on information such as: performances and capabilities of the available networks, requirements from applications, user preferences, and network operators' constraints.

However, most of the classical handover decision algorithms take into consideration only a few selection criteria, usually the Received Signal Strength (RSS) or the Signal-to-Noise Ratio (SNR). The multiple selection criteria algorithms have recently emerged as a better alternative. As a consequence, the interface selection problem (i.e., vertical handover decision) for multiinterface mobile terminals has gained importance (see [1], [2], [3]] and references therein).

Moreover, the standardization bodies, such as 3GPP, IETF and IEEE, have already commenced to tackle these issues and there are several activities ongoing. For example, the [5] describes the interworking between the 3GPP systems and IEEE 802.11 WLANs including the access network selection; then, the Candidate Access Router Discovery (CARD) draft proposed at IETF (see [6]) recognizes the need for an Access Router selection algorithm; and, finally, the IEEE 802.21 working group, e.g., see [7], works on a new standard which aims at facilitating vertical handovers based on generic triggers and on network assistance for access detection and selection.
Therefore, we reckon that the future multi-criteria handover algorithms will be more complex than nowadays as they will interact with other mechanisms in order to take into account various profiles, negotiate the QoS, or activate the networks' interfaces.

Nonetheless, exhaustive test-case scenarios for assessing existing and brand-new decision algorithms are necessary, yet these scenarios remain quite difficult to design and to implement. Accordingly, our current work concentrates on a comprehensive methodology for evaluating and comparing the inter-technologies handover algorithms; on the one hand, we suggest running these algorithms against realistic user mobility patterns for heterogeneous scenarios; and, on the other hand, we propose the definition of a common metric, namely the Standard Deviation from Optimum Interface, which we employed for algorithms' assessment.

The paper is organised as follows. We will first present the related approaches in Section 2. Then we will describe our methodology in detail in Section 3. In the next section we will introduce a new metric for comparing the vertical handover algorithms. Section 5 will present the implementation and the results obtained so far. We will reveal the future work and conclude with Section 6.

\section{User Mobility Patterns in Heterogeneous SCENARIOS}

As it has generally been admitted, a decision algorithm suggests a vertical handover whenever a "better" access network is within the terminal reach. Therefore, the behaviour of the mobile terminal is highly dependent on the user mobility patterns; thus, a basic requirement when using an assessment method is to have realistic mobility models. There are at least three different approaches which try to capture the user mobility patterns, as presented in the next paragraphs.

\section{A. Analytical methods based on the mathematical formulation of user movements}

Because many models exist in the literature (see [8] for a complete survey), we will give here just a few examples:

1) Random Waypoint Mobility Model: the terminals move independently to a random destination with a randomly 


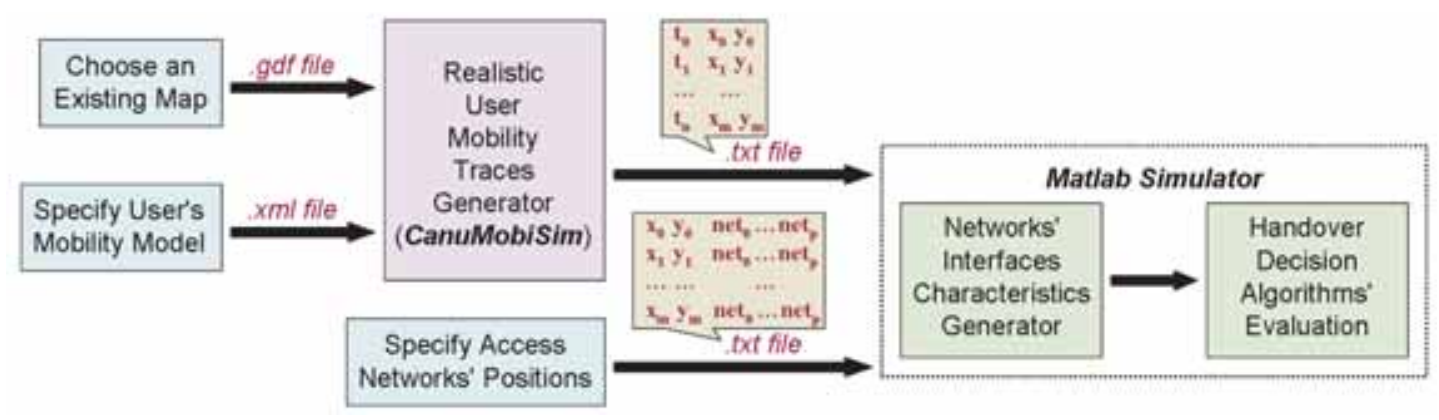

Fig. 1. Our evaluation methodology

chosen constant speed; when reaching that destination, the terminal stops for a "pause time" and it starts moving again to another destination;

2) Brownian Walk Mobility Model: similar to the Random Waypoint Model but the "pause time" is zero and the speed changes at each time interval, i.e., not only after reaching a particular destination;

3) Gauss-Markov Mobility Model: it assumes that the speed of the mobile terminal is correlated over time and modelled as a Gauss-Markov stochastic process, i.e., we have a temporal restriction of the speed;

4) Smooth Random Mobility Model: in this model both the speed and the movement direction of the terminal are decided by their previous speed and direction values, and they change incrementally and smoothly; therefore, this model captures the characteristics of a temporal restriction for both the speed and the direction;

5) Pathway Mobility Model: it integrates geographic constraints in the model by restricting the mobile terminal movements to certain pathways from a map.

However, the main drawback of all these analytical methods is the fact that the resulted mobility models are most probably unrealistic as they do not really model typical end-user's activities.

B. Measuring geographic position along with the access network capabilities in that location

It is also possible to define multi-access mobility models based on field measurements (see, e.g., [9]). Thus, for each geographical position, e.g., obtained with GPS or Galileo, the access network characteristics are measured (e.g., the available bandwidths).

Nonetheless, the user mobility models obtained with this method depend on the specific measurements done in a geographical area and on the existence of a particular access network in that area. Moreover, it might be expensive and time consuming to acquire a sufficient amount of data for the algorithms' evaluation.

C. Analysing real traces obtained from the wireless network operators

Realistic user mobility patterns can also be obtained by exploring the traces collected by some network operators. For example, the traces are gathered from base stations (i.e., for the GPRS) and from access points (i.e., for IEEE 802.11a WLAN case) and thus, the events associated with the initial connections or with the vertical handovers are captured along with the corresponding time-stamp. Then, mathematical methods are employed in order to predict and simulate the user movements; e.g., Pareto, exponential or other distributions may be found appropriate (see [10]).

However, the main drawback with this approach is that the user mobility patterns resulted from the traces obtained by a network operator are only characteristic to that specific access network and, once again, their obtainment is time consuming.

\section{The Proposed Evaluation Methodology}

Because it is very difficult (i.e., virtually impossible) to accede to the traces captured by the network operators offering services in heterogeneous environments, e.g., GPRS and IEEE 802.11a WLAN, our choice is to somehow combine the first two before-mentioned approaches: analytical models and geographic locations. Consequently, the simulation methodology we have followed is depicted in Fig.1.

Accordingly, for generating realistic user mobility patterns we have used the CanuMobiSim simulator from [11]; this simulator is fed with a real map, e.g., Brussels area from Fig.2, and with a user mobility XML file. This XML file contains the user mobility pattern which can be chosen amongst several possibilities; for instance, we have employed the following three mobility models (which are variants of Pathway Mobility Model introduced before):

- Random Pathway with Constant Speed: a user moves randomly with a constant speed, but always on the streets as provided within the map;

- Random Pathway with Smooth Motion: the same as the previous one, but the user's speed can now change with a certain acceleration/deceleration;

- Activity Based with Constant Speed and Dijkstra Path Selection: it produces itineraries according to an activity automaton when the user moves with a constant speed; the itineraries are produced between the points of interest by using Dijkstra shortest-path algorithm for the path selection.

Therefore, the CanuMobiSim simulator combines the real map constraints with the end-user mobility behaviour; the 


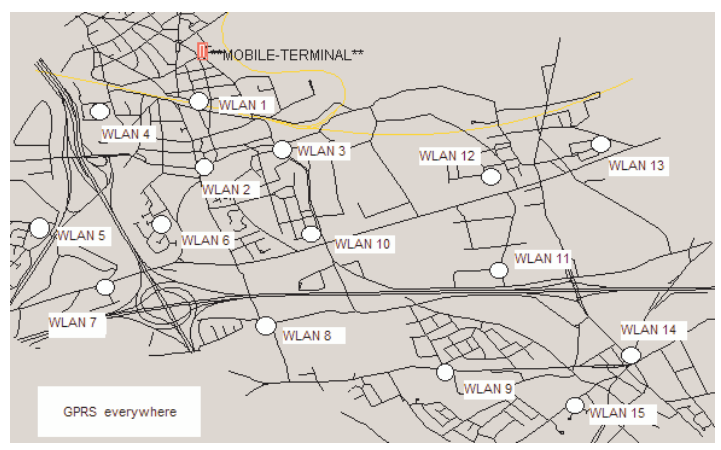

Fig. 2. Access networks' topology

output of the CanuMobiSim simulator gives the corresponding position of the mobile user at each moment in time (see Fig.1).

Furthermore, we have to build a file with the radio access points' positions; for example, the CanuMobiSim simulator can indicate the coordinates of particular points of interest, such as parking places, railway stations, commercial areas.

Thus, the file with the network positions together with the file containing the user mobility traces constitute the input for our simulator. We chose the Matlab environment because we simply wanted to have a proof of concept for two of the vertical handover algorithms; nonetheless, other simulators, such as NS-2 or Opnet, could be alternatively used.

The first component of our Matlab simulator is the network interfaces matrices generator; this component gives at each time instance the available networks and their QoS characteristics; the average performances of the GPRS network was taken from standards (see [12]), e.g., the mean throughput is set to $40 \mathrm{kbps}$; as for the IEEE 802.11a WLAN access networks a simple distance-based coverage model was used, as defined within the network interface card's specifications (e.g., [13]).

The second component of the Matlab simulator contains two vertical handover algorithms from [3] translated in Matlab. The corresponding equations for these algorithms are given in (1), (2), (3) for the weighted-sum-of-objectives algorithm and in (1), (2), (4) for the global-criterion algorithm (see also [4]).

Basically, the two algorithms calculate the access network's score functions $S$ and the application's utility functions $U$, which then are both maximized; the score is calculated for each network $i$ and the utility for each network-flow $(i, j) 2$ tuple; this multi-objective approach assumes the existence of several (in our case two) goals which can sometimes diverge, e.g., the user's score $S$ express the willingness of the end-user to favour either the QoS or the Cost, while the application's utility $U$ capture the application's requirements in terms of QoS and security.

Within these equations: $B$ is the available bandwidth, $b$ is the required bandwidth, $E$ is the bit error rate, $e$ is the acceptable bit error rate, $D$ is the average delay, $d$ is the maximum supported delay, $S$ is the security level for an access network, $s$ is the required security level, $C$ is the monetary cost, and $\gamma$ is the Cost vs. QoS goal preference. Finally, the reference values are considered as follows: $B_{\text {ref }}$ is $1 \mathrm{kbps}$, $E_{\text {ref }}$ is $10^{-2}$, $D_{\text {ref }}$ is $500 \mathrm{~ms}$, and $C_{\text {ref }}$ is 1 Euro/MB.

$$
\begin{aligned}
S(i)= & \gamma \cdot\left(\ln \frac{B_{i}}{B_{\text {ref }}}+\ln \frac{E_{\text {ref }}}{E_{i}}+\ln \frac{D_{\text {ref }}}{D_{i}}\right) \\
& +(1-\gamma) \cdot \ln \frac{C_{i}}{C_{r e f}} ; \\
U(i, j)= & \ln \frac{B_{i}-b_{j}}{B_{r e f}}+\ln \frac{e_{j}-E_{i}}{E_{r e f}} \\
& +\ln \frac{d_{j}-D_{i}}{D_{r e f}}+\ln \frac{S_{i}-s_{j}}{S_{r e f}}
\end{aligned}
$$

$$
\begin{gathered}
C(i, j)=0.5 \cdot S(i)+0.5 \cdot U(i, j) \\
C(i, j)=\left(\frac{S_{\text {ideal }}-S(i)}{S_{\text {ideal }}}\right)^{2}+\left(\frac{U_{\text {ideal }}-U(i, j)}{U_{\text {ideal }}}\right)^{2} .
\end{gathered}
$$

To conclude, we underline the fact that both algorithms' outcomes will contain an ordered list of preferred network interface $i$, for each application flow $j$, based on the scoreutility combinations $C(i, j)$.

\section{STANDARD DEVIATION FROM OPTIMUM INTERFACE METRIC}

As far as we know, and let aside the common quantitative metrics such as algorithm's execution speed, there are no qualitative metrics for vertical handover algorithms' evaluation; thus, we had to define a new one. Based on the well-known standard deviation (i.e., which calculates the deviation from an average value), we have defined a novel metric called Standard Deviation from Optimum Interface (SDOI).

Let us assume that we have $n$ network interfaces within our mobile terminal, and $i$ from 1 to $n$. First, we introduce the optimum interface definition in (5); the optimum interface can be seen as an ideal interface (i.e., a virtual interface). The notations are the same as those used in the previous equations.

$$
O=\left[\max B_{i}, \min E_{i}, \min D_{i}, \min C_{i}, \max S_{i}\right]
$$

Then, we define the standard deviation from optimum for a particular decision parameter (e.g., bandwidth) in (6), where $x_{i, j}$ is the value for parameter $j$ at time instance $i$ for the proposed interface (as chosen by the handover algorithm), and $\bar{x}_{i, j}$ is the value for parameter $j$ at time instance $i$ for the optimum interface $O$.

$$
s d o_{j}=\sqrt{\left(\sum_{i=1}^{m}\left(x_{i, j}-\bar{x}_{i, j}\right)^{2}\right) / m} ;
$$



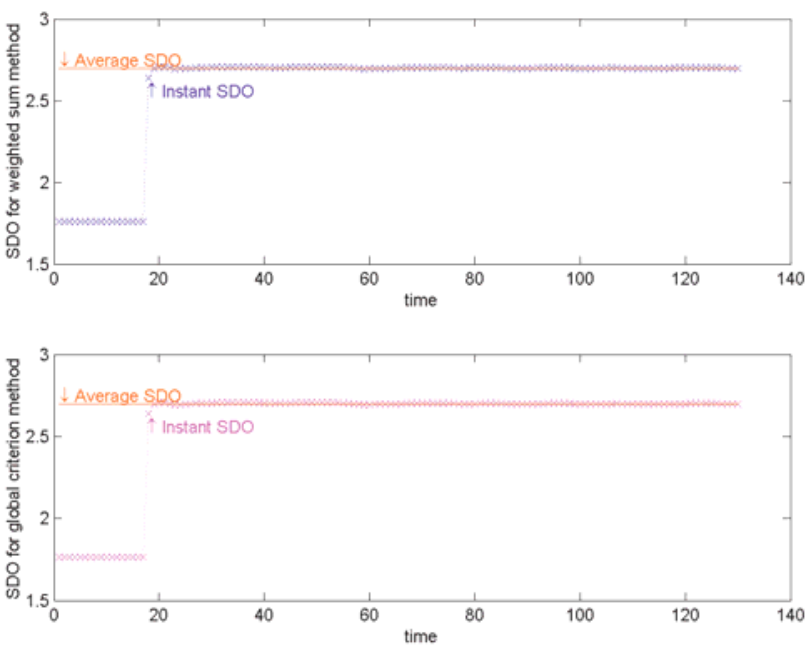

Fig. 3. Simulation results for the SDOI metric

Eventually, we can now give the formula for the new SDOI metric in (7); the logarithm is needed to make meaningful the sum of particular deviations with different measurement units.

$$
S D O I=\sum_{j=1}^{p} \ln \left(s d o_{j}\right) .
$$

Thus, the SDOI metric defines the global deviation from the optimum interface for all decision parameters used within a vertical handover decision algorithm.

\section{Simulation Results}

We have simulated a mobile terminal in the Brussels area which is within the coverage of two access networks: GPRS and WLAN; the three above-mentioned user mobility models were employed; the locations of the 15 WLANs were kept unchanged in all models. Furthermore, the vector with application's demands is considered as being: required bandwidth $b=32 k b p s$, acceptable bit error rate $e=10^{-4}$, maximum supported delay $d=3 \mathrm{~ms}$, and required security level $s=1$.

The Fig. 3 shows the SDOI metric's results obtained from the simulation done for the specified weighted-sum-of-objectives and global-criterion methods; a random pathway with constant speed mobility model was used for generating the results presented in this figure.

It can be pointed out that the results from the SDOI metric are similar for both decision algorithms regardless of the three user mobility models used in our simulation. This means that the handover decisions generated by the two algorithms are the same for the simulated cases.

\section{CONCLUSIONS AND FUture WORK}

We argue that the vertical handover decision algorithms within the future heterogeneous wireless networks will be much more complex than nowadays.
Therefore, the main goals of this paper were to introduce a comprehensive methodology for assessing the intertechnologies handover algorithms and to provide a common metric when comparing the algorithms' performances. The proposed methodology combines the analytically-obtained mobility models with the geographic locations of the radio access networks and their capabilities. Moreover, the builtin modules can be separately improved, and the novel SDOI metric can be easily extended to encompass more decision parameters.

The envisaged future work will consists in extending the scenarios to several mobile terminals and in using different maps and different mobility patterns. In addition, the network performances need to be simulated more accurately by taking into account not only the distance-based coverage models but also the radio channel characteristics, such as received signal strength (RSS), interferences, or fading.

Finally, as both handover decision algorithms were fully end-terminal oriented, in our simulation we have used only one mobile node; if we had used more mobile terminals, we would have also needed the collaboration of access networks, e.g., if several terminals had tried to switch on the same access network at the same moment, we would have had to interact with some admission control mechanisms; nevertheless, the development and the implementation of such mechanisms is considered as future work.

\section{REFERENCES}

[1] W. Zhang et al., Design and Evaluation of a Handover Decision Strategy for 4th Generation Mobile Networks, 57th Semi-annual Vehicular Technology Conference VTC, April 2003

[2] M. O'Droma et al., "Always Best Connected" Enabled 4G Wireless World, IST Mobile and Wireless Communications Summit 2003, June 2003

[3] L. Suciu et al., Achieving "Always Best Connected" through Extensive Profile Management, in Proc. of 9th Int. PWC Conference, Sept. 2004

[4] Y. Collette and P. Siarry, Multiobjective Optimization - Principles and Case Studies, Springer, August 2003

[5] 3GPP TS 23.234, 3GPP system to Wireless Local Area Network (WLAN) Interworking, System Description (Release 6) V2.3.0, November 2003

[6] M. Liebsch and A. Singh (Editors), Candidate Access Router Discovery, RFC 4066, July 2005

[7] M. Williams, Directions in Media Independent Handover, IEICE Transactions on Fundamentals of Electronics, Communications and Computer Sciences - Special Section on Multi-dimensional Mobile Information Networks, July 2005

[8] T. Camp et al., A Survey of Mobility Models for Ad Hoc Network Research, Wireless Communications and Mobile Computing (WCMC 2002): Special issue on Mobile Ad Hoc Networking: Research, Trends and Applications

[9] Juuso Pesola et al., Location-Aided Handover in Heterogeneous Wireless Networks, Wireless Personal Communications, Special Issue on Cellular and Wireless Location Based Technologies and Services, Volume 30, 2004

[10] S. Thajchayapong and J.M. Peha, Mobility patterns in microcellular wireless networks, IEEE Wireless Communications and Networking, 2003, Volume: 3 , 16-20 March 2003

[11] I. Stepanov et al., A Meta-Model and Framework for User Mobility in Mobile Networks, In Proceedings of the 11th International Conference on Networking 2003 (ICON 2003), October 2003

[12] 3GPP, Digital Cellular Telecommunications System (Phase 2+) (GSM); General Packet Radio Service (GPRS); Service description; Stage 2, Version 7.7.0, Release 1998

[13] Atheros Communications Inc., Measured Performance of 5-GHz $802.11 \mathrm{a}$ Wireless LAN Systems, White Paper, August 2001 